AIAA 2002-2773

\title{
ROUND TURBULENT PUFFS AND BUOYANT THERMALS IN UNIFORM CROSSFLOWS
}

\author{
F.J. Diez, ${ }^{*}$ O.C. Kwon, ${ }^{\dagger}$ R. Sangras, ${ }^{\ddagger}$ L.P. Bernal, ${ }^{* *}$ G.M. Faeth ${ }^{\dagger}$ \\ Department of Aerospace Engineering \\ The University of Michigan \\ Ann Arbor, MI 48109-2140
}

\begin{abstract}
The properties of round nonbuoyant turbulent puffs, and buoyant turbulent thermals in uniform crossflows were studied both experimentally and theoretically, motivated by applications to interrupted gas and liquid releases caused by process upsets, explosions and unwanted fires, among others. Emphasis was placed on self-preserving conditions far from the source where extraneous source disturbances have been lost, where flow properties are largely controlled by the conserved properties of the flow, and where properly scaled flow properties become independent of both distance from the source and time. The experiments involved injecting dye-containing turbulent round water puffs and thermals into a uniform crossflow produced by a 610 x $610 \mathrm{~mm}$ crosssection water tunnel facility and observing the flow with CCD cameras. Near-source properties varied significantly with source properties but the flows became turbulent and then self-preserving within 5 and 30-40 source diameters in the vertical direction (aligned with the source puff/thermal) from the source, respectively. Within the self-preserving region, the vertical penetration distance generally varied as a function of time in accord with self-preserving predictions. Flow in the self-preserving region, also satisfied the assumption of no slip crosstream convection at the mean crosstream velocity, yielding a simple and convenient way of predicting flow trajectories. Radial penetration distances of the flows also satisfied the general scaling relationships for puffs and thermals in still fluids but with the added complication that the flow was no longer axisymmetric
\end{abstract}

* Post-Doctoral Research Fellow, Department of Aerospace Engineering, AIAA member.

$\dagger$ Now at University of Southern California, Los Angeles, CA.

* Now at Worcester Polytechnic Institute, Worcester, MA.

** Associate Professor, AIAA member.

i† A.B. Modine Professor, Department of Aerospace Engineering, Fellow AIAA, Corresponding author, Tel.: +1-734-764-7202;

Fax:+1-734-936-0106; E-mail: gmfaeth@umich.edu (G.M. Faeth) Copyright (C) 2002 by G.M. Faeth. Published by the American Institute of Aeronautics and Astronautics, Inc., with permission. about the axis of the trajectory. Finally, when amounts of source fluid injected became large for puffs and thermals, self-preserving behavior for starting jets and plumes was achieved before the source flow was terminated and the flow was best considered to be an interrupted jet or plume rather than a puff or a thermal.

\section{NOMENCLATURE}

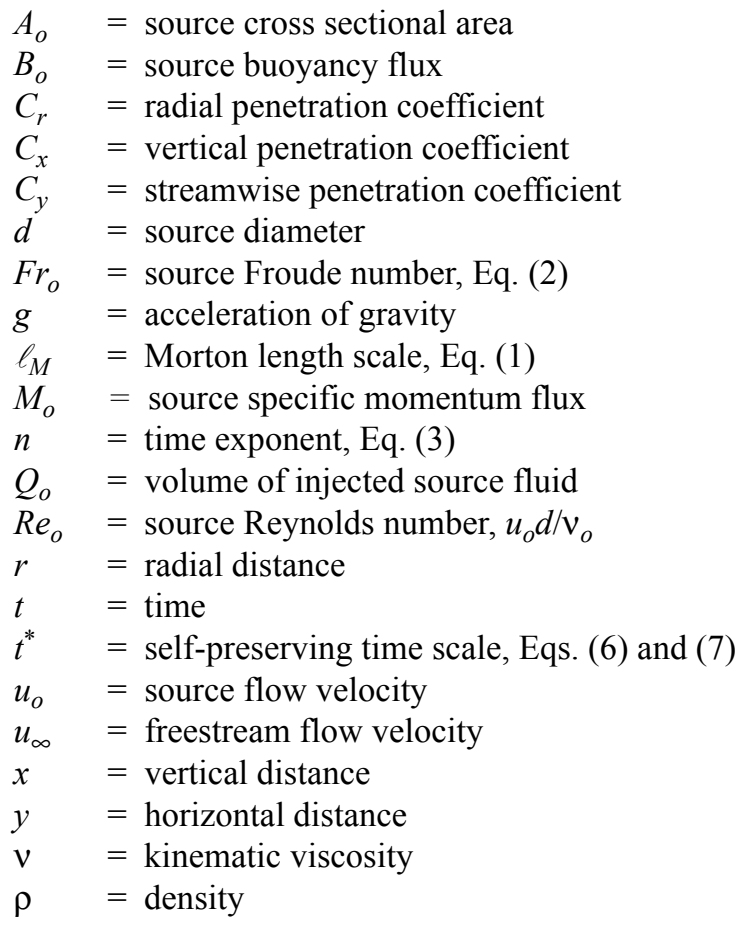

Subscripts

$\mathrm{d} \quad=$ delay

$\max =$ maximum value

$\mathrm{p} \quad=$ maximum penetration location

$\mathrm{o} \quad=$ initial or virtual origin value

$\infty \quad=$ ambient value 


\section{INTRODUCTION}

Past experimental, theoretical and computational studies of round turbulent nonbuoyant puffs (puffs) and buoyant thermals (thermals) have been reported by a number of workers. ${ }^{1-10}$ For example, classical studies of puffs and thermals in still environments have been reported by Rouse et al., ${ }^{3}$ Morton et al., ${ }^{4}$ Morton, ${ }^{5}$ and Scorer ${ }^{6}$ who developed turbulent scaling rules that help to summarize the main features of these flows. Difficulties with these studies, however, involve concerns that experimental observations were not carried out far enough from the source for self-preserving turbulent flow behavior to be achieved and limited experimental observations of puffs and thermals. The recent studies of Sangras et al. ${ }^{1}$ and Diez et al. ${ }^{2}$ addressed these issues for puffs, starting jets, thermals and starting plumes in still environments, finding that the self-preserving penetration properties of the flows was achieved at distances greater than 20-30 source diameters from the source and providing subsequent measurements of streamwise and radial penetration properties of the flows for self-preserving conditions.

Experimental, theoretical and computational studies of puffs and thermals in uniform crossflows generally have been more recent than studies of puffs and thermals in still environments; some typical examples are studies reported by Andreopoulos, ${ }^{7}$ Alton et al., ${ }^{8}$ Baum et al. ${ }^{9}$ and Hasselbrink and Mungal. ${ }^{10}$ These studies also involve concerns about whether observations were carried out far enough from the source for self-preserving conditions to be reached; in addition, available information about puffs and thermals in crossflows is very sparse. Based on these observations, the objectives of the present investigation were to consider round turbulent nonbuoyant puffs and buoyant thermals in uniform crossflows, as follows: (1) to measure the streamwise and crosstream penetration properties of these flows as functions of time for various source diameters, source Reynolds numbers, source Froude numbers, source/ ambient density ratios, source/ambient characteristic velocity ratios, and amounts of injected source fluid; and (2) to use the measured penetration properties to evaluate self-preserving scaling and determine the empirical factors needed to correlate the streamwise, crosstream and radial penetration properties of these flows.

\section{EXPERIMENTAL METHOD}

\section{Apparatus}

The experiments involved fresh water modeling of turbulent nonbuoyant flows and salt water modeling of turbulent buoyant flows, the last as suggested by Steckler et al. ${ }^{11}$ These experiments were carried out in a water tunnel facility with a test section having a $610 \times 610 \mathrm{~mm}$ cross section and a streamwise length of roughly 2000 $\mathrm{mm}$. A sketch of this facility appears in Fig. 1. The water tunnel was a closed loop arrangement with water entering the test section through a calming section, a flow straightener grid, a series of screens and a 10:1 contraction to provide a small turbulence intensity $(<$ $1 \%)$ mean flow through the test section. The test section had plexiglass walls (16 mm thick) to provide optical access. The water in the water tunnel was obtained from the laboratory water supply but was heated to room temperature before reaching the water tunnel so that it was readily degassed of dissolved air by agitation. For thermals, the dense salt-containing source liquid settled naturally to the bottom of the water tunnel and was removed from time to time using a drain.

The puffs and thermals were injected into the top of the test section through smooth round tubes, with fullydeveloped turbulent pipe flow at the tube exit, using syringe pumps that could be programmed to start, stop and deliver puff and thermal liquid at prescribed rates. The dye-containing source liquid was illuminated and photographed using color video cameras with these records subsequently analyzed to obtain puff and thermal trajectories and dimensions as a function of time.

\section{Test Conditions}

Present test conditions for round turbulent nonbuoyant puffs and buoyant thermals will be summarized on plots of the data. Test conditions involved source diameters of 3.2 and $6.4 \mathrm{~mm}$, source/ambient density ratios of 1.000 , 1.073 and 1.150, source Reynolds numbers of 4,00011,000, source Froude numbers of 14-82 (for thermals), amounts of source fluid injected for puffs and thermals of 16-318 source diameters, vertical penetration distances up to 200 source diameters and ratios of source flow velocities to crosstream flow velocities of 5-35.

\section{THEORETICAL METHODS}

Two parameters that are useful for estimating when turbulent flows from interrupted sources become self-preserving are the distance from the virtual origin normalized by the source diameter, $\left(x-x_{o}\right) / d$, and dis- 
tance from the virtual origin normalized by the Morton length scale $\left(x-x_{o}\right) / l_{M}$, see Dai et al. ${ }^{12}$ The first parameter is pertinent to both nonbuoyant and buoyant flows and measures the distance needed to modify distributions of mean and fluctuating properties from conditions within a source passage to conditions in an unbounded flow (e.g., to eliminate source disturbances). The second parameter is only pertinent to buoyant flows and measures the distance required for buoyancy-induced momentum to become large compared to the source momentum so that the buoyant features of the flow are dominant. The Morton length scale is defined as follows for round buoyant sources having uniform properties: ${ }^{5,13}$

$$
\ell_{\mathrm{M}} / \mathrm{d}=(\pi / 4)^{1 / 4}\left(\rho_{\infty} \mathrm{u}_{\mathrm{o}}^{2} /\left(\mathrm{gd}\left|\rho_{\mathrm{o}}-\rho_{\infty}\right|\right)\right)^{1 / 2}
$$

where an absolute value has been used for the density difference in order to account for both rising and falling flows. The source Froude number, $F r_{o}$, is proportional to $l_{M} / d$ for uniform source properties, as follows: ${ }^{12}$

$$
\operatorname{Fr}_{\mathrm{o}}=(4 / \pi)^{1 / 4} \ell_{\mathrm{M}} / \mathrm{d}
$$

The Froude number is often used to characterize the initial degree of buoyant behavior of the source, e.g., Fr $=$ 0 , and $\infty$ for purely buoyant and purely nonbuoyant sources, respectively. Past studies of steady round turbulent buoyant plumes suggests $\left(x-x_{o}\right) / d>80$ and $\left(x-x_{o}\right) / \ell_{M}$ $>10$ for self-preserving conditions, see Dai et al. ${ }^{12}$ and references cited therein. Another important criterion for self-preserving flows is that properties within the flow should not be very different from ambient properties, so that property changes are nearly linear functions of the degree of mixing. For present flows, where the main property variation involves the density, this last criterion for self-preserving behavior implies $\left|\rho_{0}-\rho_{\infty}\right| / \rho_{\infty} \ll 1$. Present test conditions are limited to maximum values of $\left|\rho_{o}-\rho_{\infty}\right| / \rho_{\infty}=0.150$; therefore, this last criterion is generally satisfied.

Present theory involved exploiting concepts of self-preserving flow to predict (correlate) puff and thermal trajectories and dimensions as a function of time. This simplified approach was carried out under the same general approximations as the detailed numerical simulations of steady plumes in crossflow reported by Baum et al. ${ }^{9}$ In particular, streamwise motion of the flow was assumed to involve simple convection in the uniform crossflow with negligible slip, which is identical to the approach of Baum et al. ${ }^{9}$ Rather than numerically com- pute the vertical rise of the flow, however, the temporal similarity scaling of starting plumes and thermals was used, see Diez et al. ${ }^{2}$ for these results.

Assuming that the flow is within the self-preserving region, expressions for the streamwise penetration distance of the present puff and thermal flows are available from past work, see Morton et al., ${ }^{4}$ Scorer, ${ }^{6}$ List, ${ }^{13}$ Turner, ${ }^{14-16}$ Batt et al., ${ }^{17}$ Glezer and Coles, ${ }^{18}$ Shariff and Leonard, ${ }^{19}$ and references therein. The configurations of the present buoyant and nonbuoyant flows for these analyses are sketched in Fig. 2. The source flow enters from a passage having a diameter, $d$, with a density, $\rho_{\mathrm{o}}$, and velocity $u_{\mathrm{o}}$, and flows into an environment having a density, $\rho_{\infty}$, and a uniform crossflow velocity of $\mathrm{u}_{\infty}$; properties of interest include the maximum vertical, crossflow and radial penetration distances, $x_{\mathrm{p}}, y_{\mathrm{p}}$ and $r_{\mathrm{p}}$, illustrated in Fig. 2. The main difference between the two flows is that $g=0$ for the nonbuoyant flows. Major assumptions for present considerations of selfpreserving flow properties are similar to earlier analyses to find self-preserving flow scaling: physical property variations in the flows are assumed to be small; the present interrupted sources start and stop instantly and maintain constant source properties during the period of flow as already noted, the crosstream motions of the flow approximate no-slip convection at the uniform ambient crossflow velocity, virtual origins were used to maximize conditions where self-preserving behavior is observed, and (for convenience) source properties are assumed to be uniform similar to the conditions required for Eqs. (1) and (2). The last approximation is not a critical assumption, however, because the details of the sources are not important for self-preserving flows, whereas conserved properties of the flows are adequately prescribed by mean source properties, within the self-preserving regions of the present flows.

Under these assumptions, the temporal variation of the maximum vertical penetration distance can be expressed as follows within the self-preserving regions of the present unsteady turbulent flows:

$$
\left(x_{p}-x_{o}\right) / d=C_{x}\left(\left(t-t_{d}\right) / t^{*}\right)^{n}
$$

The corresponding temporal variation of the maximum radial penetration distance can be expressed most conveniently in terms of the vertical penetration distance, as follows:

$$
\mathrm{r}_{\mathrm{p}} /\left(\mathrm{x}_{\mathrm{p}}-\mathrm{x}_{\mathrm{o}}\right)=\mathrm{C}_{\mathrm{r}}
$$


Based on the no-slip convection approximation, the horizontal penetration distance is:

$$
\left(\mathrm{y}_{\mathrm{p}}-\mathrm{y}_{\mathrm{o}}\right) / \mathrm{d}=\mathrm{C}_{\mathrm{y}}\left(\mathrm{u}_{\infty}\left(\mathrm{t}-\mathrm{t}_{\mathrm{d}}\right) / \mathrm{d}\right)
$$

The values of $C_{x}, t^{*}, n, C_{r}$ and $C_{y}$ vary depending upon the particular starting flow that is being considered. The values of $C_{r}$ and $C_{y}$ are best fit empirical parameters of the self-preserving analyses which will be considered later when the measurements are discussed. The values of $t^{*}$ and $n$, however, follow from the requirements for self-preserving flows and can be expressed as follows for the present unsteady flows:

$$
\begin{gathered}
\mathrm{t}^{*}=\mathrm{d}^{4} / \mathrm{M}_{\mathrm{o}}, \mathrm{n}=1 / 4 \text {; nonbuoyant puff } \\
\mathrm{t}^{*}=\left(\mathrm{d}^{4} / \mathrm{B}_{\mathrm{o}}\right)^{1 / 2}, \mathrm{n}=1 / 2 ; \text { buoyant thermal }
\end{gathered}
$$

where $\mathrm{M}_{\mathrm{o}}=\mathrm{Q}_{0} \mathrm{u}_{\mathrm{o}}$ is the source specific momentum flux, and $\mathrm{B}_{\mathrm{o}}=\mathrm{Q}_{0} \mathrm{~g}\left|\rho_{\mathrm{o}}-\rho_{\infty}\right| / \rho_{\infty}$ is the source buoyancy flux of a thermal and an absolute value has been used for the density difference, as before, to account for both rising and falling flows. The expressions for vertical penetration distance of puff and thermals in a crossflow, Eqs. (3), (6) and (7), are convenient for illustrating the development of puffs and thermals toward self-preserving behavior, and their subsequent penetration properties in the self-preserving portion of the flow. This formulation is misleading, however, because it involves the source diameter which is not a relevant variable of self-preserving flows. This is apparent because $d$ cancels out of Eqs. (3), (6) and (7), to yield the following expressions for the vertical penetration distance of a self-preserving puff and thermal in a crossflow:

$$
\begin{aligned}
& \left(\mathrm{x}_{\mathrm{p}}-\mathrm{x}_{\mathrm{o}}\right) /\left(\mathrm{M}_{\mathrm{o}}\left(\mathrm{t}-\mathrm{t}_{\mathrm{d}}\right)\right)^{1 / 4}=\mathrm{C}_{\mathrm{x}} ; \text { nonbuoyant puff } \\
& \left(\mathrm{x}_{\mathrm{p}}-\mathrm{x}_{\mathrm{o}}\right) /\left(\mathrm{B}_{\mathrm{o}}^{1 / 2}\left(\mathrm{t}-\mathrm{t}_{\mathrm{d}}\right)\right)^{1 / 2}=\mathrm{C}_{\mathrm{x}} ; \text { buoyant thermal }
\end{aligned}
$$

where the theoretical value, $n=1 / 4$ and $1 / 2$, for self-preserving puffs and thermals has been used in Eqs. (8) and (9) respectively.

\section{RESULTS AND DISCUSSION}

Facility Evaluation

Initial measurements in the water tunnel illustrated in Fig. 1 involved characterizing the water tunnel flow using hot-film anemometer. The flow was found to be properly uniform with a small turbulence intensity (noted earlier) in the region where the puffs and thermals were present.

Results for puffs and thermals are discussed in the following. Findings for puffs and thermals include visualization of the flow; correlation of measured horizontal penetration distances as function of time with the scaling law of Eq. (5); correlation of measured vertical penetration distances as a function of time with the appropriate self-preserving scaling law of Eqs. (3), (6) and (7); and correlation of the measured maximum radial penetration distances as a function of time with the self-preserving scaling law of Eq. (4). Various cross correlations of horizontal, vertical and radial penetration distances were also considered. The ranges of $\left(x_{\mathrm{p}}-x_{\mathrm{o}}\right) / d$ and the values of $C_{x}, t^{*}, n, C_{r}$ for round turbulent nonbuoyant puffs and buoyant thermals are summarized in Table 1.

\section{$\underline{\text { Puffs }}$}

Video images of a typical round nonbuoyant turbulent puff at various times after initiation of the flow are illustrated in Fig. 3. The appearance of the flow in the early stages (not shown in Fig. 3) when the source flow is maintained is naturally identical to the starting jet. When the source flow is terminated, however, the leading turbulent vortex and the trailing starting jet portions of the flow continue to penetrate into the cross-stream liquid with a thin stem connecting the base of the interrupted jet to the source. The main difference between the motion of puff and a thermal is that the former has a much smaller velocity, which is evident from the different scaling rules of puffs and thermals indicated by Eqs. (3),(6) and (7), e.g., $x_{\mathrm{p}} \sim t^{\mathrm{n}}$ with $n=1 / 4$ and $1 / 2$ respectively for puffs and thermals. An interesting behavior of the puff is that it is affected by the amount of source fluid used to form the puff. Large amounts of source fluid yield a leading vortex followed by an extended trailing jet-like region. Reduced amounts of source fluid tend to reduce the extent of the trailing jet-like region, see Scorer ${ }^{6}$. In addition, continued penetration of the puff implies continued increases of the volume of the leading vortex; this causes the trailing jet to eventually be engulfed by the leading vortex followed by continued dilution of the leading vortex. Thus, there is significant potential for several different flow regimes during both the development and eventual self-preserving flow periods of the life time of a puff.

Normalized horizontal penetration distances of puffs are plotted according to the no-slip convection of Eq. (5) in Fig. 4. The results indicate that this assumption is satis- 
fied for the range of the measurements, e.g., $\mathrm{u}_{\infty}\left(\mathrm{t}-\mathrm{t}_{\mathrm{d}}\right) / \mathrm{d}>50$.

Normalized vertical penetration distances of puffs are plotted according to the self-preserving scaling of Eqs. (3), and (6) in Figs. 5 and 6, as functions of dimensionless time and dimensionless cross stream distance, respectively. Present measurements indicate that selfpreserving scaling for puffs occurs at $\left(t-t_{\mathrm{d}}\right) u_{\mathrm{o}} / d>300$, and $\left(x_{\mathrm{p}}-x_{\mathrm{o}}\right) / d>30-40$, which agrees with earlier observations of starting puffs in still fluids due to Sangras et al. ${ }^{1}$

Normalized maximum radial penetration distances for puffs are plotted according to the self-preserving scaling of Eq. (4) in Fig. 7 as functions of dimensionless vertical penetration distance. The normalized radial penetration distance has relatively small values in the region nearest the source, specially for large diameter sources, e.g. $d=6.4 \mathrm{~mm}$; such behavior is expected, however, because the flow has not reached the self-preserving region and the relatively large negative virtual origin calculated allows the normalized radial penetration to increase first with increasing streamwise distance before becoming relatively constant in the self-preserving region where $\left(x_{\mathrm{p}}-x_{\mathrm{o}}\right) / d>70-80$. For self-preserving conditions, $r_{\mathrm{p}} /\left(x_{\mathrm{p}}-x_{\mathrm{o}}\right)=C_{\mathrm{r}}=0.14$ which is shown in Fig. 7 for reference purposes. In addition, the normalized expression for the vertical penetration distance from Eq. (8) is illustrated in Fig. 7; as already noted, vertical penetration for thermals in crossflow reaches self-preserving behavior sooner than the radial penetration, e.g., at $\left(x_{\mathrm{p}}-x_{\mathrm{o}}\right) / d>40-60$ rather than $\left(x_{\mathrm{p}}-x_{\mathrm{o}}\right) / d>70-80$.

Finally, the location of the virtual origins are plotted as a function of the volume of injected fluid represented by the number of passage lengths of injected fluid, $Q_{\mathrm{o}} /$ $\left(A_{\mathrm{o}} d\right)$, in Fig. 8. All data for puffs obtained during the present investigation are shown on this figure. The results show that there is little effect of the injected fluid volume on the location of the virtual origin. These results also indicate that the virtual origin is approximately $x_{\mathrm{o}} / d=-23$ for the conditions of the present measurements.

\section{Thermals}

Video images of a typical round turbulent buoyant thermal at various times after initiation of the flow are illustrated in Fig. 9. The appearance of the thermal in the early stages, when the source flow is maintained, is naturally identical to a starting plume. Once the source flow is terminated, however, the leading turbulent vortex and the trailing plume continue to penetrate in the vertical direction. Similar to the puffs, continued pene- tration of the thermal in the vertical direction implies continued increases of the volume of the leading vortex; this causes the trailing plume to be engulfed by the leading vortex followed by continued dilution of the leading vortex. Nevertheless, the general scaling behavior of thermals did not exhibit significant changes when the leading vortex engulfed the trailing plume, similar to the behavior of puffs discussed earlier.

Normalized horizontal penetration distances of thermals are plotted according to the no-slip convection assumption of Eq. (5) in Fig. 10. The results indicate that this assumption is satisfied for the range of the measurements, which involves crosstream distances extending from near zero, similar to the results for puffs discussed in connection with Fig. 4.

Normalized vertical penetration distances of thermals are plotted according to the self-preserving scaling of Eqs. (3), and (7) in Figs. 11 and 12. Present measurements indicate that self-preserving scaling of thermals occurs at $\left(t-t_{\mathrm{d}}\right)\left(B_{\mathrm{o}} / d^{4}\right)^{1 / 2}>3000$, and $\left(x_{\mathrm{p}}-x_{\mathrm{o}}\right) / d>40-60$. The different powers of the temporal growth of thermals compared to the puffs, $n=1 / 2$ compared to $1 / 4$, results in a much faster growth rate in the vertical direction of thermals compared to puffs due to the additional buoyancy-induced momentum for thermals.

Normalized maximum radial penetration distances for thermals are plotted according to the self-preserving scaling of Eq. (4) in Fig. 13 in terms of dimensionless time and dimensionless vertical penetration distance, respectively. The behavior here is qualitatively very similar to results for puffs illustrated in Figs. 7. It turns out that the normalized maximum radial penetration distance in the self-preserving region is the same for puffs and thermals, e.g., $r_{\mathrm{p}} /\left(x_{\mathrm{p}}-x_{\mathrm{o}}\right)=C_{\mathrm{r}}=0.14$. The main difference is that the self-preserving region is reached at a later stage for thermals than puffs, e.g., $\left(x_{\mathrm{p}}-x_{\mathrm{o}}\right) / d>100$ 120 compared to 70-80. These radial penetrations for puffs and thermals in a crossflow are comparable to results for puffs in still fluid from Sangras et al. ${ }^{1}$, e.g., $C_{\mathrm{r}}$ $=0.18$, and thermals in still fluid from Diez et al. ${ }^{2}$, e.g., $C_{\mathrm{r}}=0.19$. In addition, the normalized expression for the vertical penetration distance from Eq. (9) is illustrated in Fig. 13; as already noted, vertical penetration for thermals in crossflow reaches self-preserving behavior much sooner than the radial penetration, e.g., at $\left(x_{\mathrm{p}}-x_{\mathrm{o}}\right) / d$ $>40-60$ rather than $\left(x_{\mathrm{p}}-x_{\mathrm{o}}\right) / d>100-120$.

The location of the virtual origins for thermals are plotted as a function of the volume of injected fluid represented by the number of passage lengths of injected fluid, $Q_{\mathrm{o}} /\left(A_{\mathrm{o}} d\right)$, in Fig. 14. The behavior here is qualita- 
tively similar to results for puffs; the main difference is that the mean virtual origin is slightly nearer to the source exit for thermals than for puffs, e.g., $x_{\mathrm{o}} / d=-20$ compared to -23 .

\section{CONCLUSIONS}

The temporal development of round turbulent nonbuoyant puffs and buoyant thermals in a turbulent uniform crossflow were studied both theoretically and experimentally. Conditions far from the source were emphasized where effects of source disturbances are lost, where the conserved properties for the flow control flow structure and where self-preserving behavior is approximated. Test conditions involved source diameters of 3.2 and $6.4 \mathrm{~mm}$, source/ambient density ratios of 1.000 , 1.073 and 1.150 , source Reynolds numbers of 4,00011,000, source Froude numbers of 14-82 (for thermals), amounts of source fluid injected for puffs and thermals of 16-318 source diameters, vertical penetration distances up to 200 source diameters and ratios of source flow velocities to crosstream flow velocities of 5-35.

The present flows generally became turbulent within 5 vertical source diameters from the source and became self-preserving at distances greater than 70 source diameters and 100 source diameters from the source for puffs and thermals, respectively.

Within the self-preserving region, vertical penetration distances generally varied as a functions of time in accord with self-preserving predictions. This implied vertical penetration distances increasing as a function of time to the following powers: $1 / 4$ for puffs and $1 / 2$ for thermals.

Within the self-preserving region, the assumption of noslip crosstream convection at the mean crosstream velocity also proved to be satisfactory, yielding a simple and convenient way of predicting flow trajectories.

Radial penetration distances of the flows also satisfied the general scaling relationships observed for puffs and thermals in still fluids but with the added complication that the flow was no longer axisymmetric about the axis of the trajectory. This implies the following normalized values of maximum flow radius at self-preserving conditions, e.g., $r_{\mathrm{p}} /\left(x_{\mathrm{p}}-x_{\mathrm{o}}\right)=0.14$ for both puffs and thermals.

Finally, the results show that there is little effect of the injected fluid volume in the location of the virtual origin, e.g., for the amounts injected the virtual origin was approximately $x_{\mathrm{o}} / d=-23$ and -20 for puffs and thermals, respectively.

\section{ACKNOWLEDGEMENTS}

This research was supported by the United States Department of Commerce, National Institute of Standard and Technology, Grant Nos. 60NANB8D0081 and 60NANB1D0006, with H. R. Baum of the Building and Fire Research Laboratory serving as Scientific Officer.

\section{REFERENCES}

${ }^{1}$ Sangras, R., Kwon, O.C., and Faeth, G.M., "Self-Preserving Properties of Unsteady Round Nonbuoyant Turbulent Starting Jets and Puffs in Still Fluids," J. Heat Trans., in press.

${ }^{2}$ Diez, F.J., Kwon, O.C., Sangras, R., and Faeth, G.M., "Self-Preserving Properties of Unsteady Round Turbulent Buoyant Plumes and Thermals in Still Fluid," J. Heat Trans., submitted.

${ }^{3}$ Rouse, H., Yih, C.S., and Humphreys, H.W., "Gravitational Convection from a Boundary Source," Tellus, Vol. 4, 1952, pp. 201-210.

${ }^{4}$ Morton, B.R., Taylor, G., and Turner, J.S., "Turbulent Gravitational Convection from Maintained and Instantaneous Sources," Proc. Roy. Soc. London A234, 1956, pp. 1-23.

${ }^{5}$ Morton, B.R., "Forced Plumes," J. Fluid Mech., Vol. 5, 1959, pp. 151-163.

${ }^{6}$ Scorer, R.S., "Experiments on Convection of Isolated Masses of Buoyant Fluid," J. Fluid Mech., Vol. 2, 1957, pp. 583-594.

${ }^{7}$ Andreopoulos, J., "Heat-Transfer Measurements in a Heated Jet-Pipe Flow Issuing into a Cold Cross Stream," Phys. Fluids, Vol. 26, No. 4, 1983, pp. 32003210 .

${ }^{8}$ Alton, B.W., Davidson, G.A., and Slawson, P.R., "Comparison of Measurements and Integral Model Predictions of Hot Water Plume Behavior in a Cross-Flow," Atmos. Environ. A-Gen., Vol. 27, No. 4, 1993, pp. 589598.

${ }^{9}$ Baum, H.R., McGrattan, K.B., and Rehm, R.G., "Simulation of Smoke Plumes from Large Pool Fires," Proceedings of the Combustion Institute, Vol. 25, 1994, pp. 1463-1469. 
${ }^{10}$ Hasselbrink, E.F., and Mungal, M.G., "Observations on the Stabilization Region of Lifted Non-Premixed Methane Transverse Jet Flames," Proceedings of the Combustion Institute, Vol. 27, 1998, pp. 1167-1173.

${ }^{11}$ Steckler, K.D., Baum, H.R., and Quintiere, J.G., "Salt Water Modeling of Fire Induced Flows in Multicomponent Enclosures," Proceedings of the Combustion Institute, Vol. 21, 1986, pp. 143-149.

${ }^{12}$ Dai, Z., Tseng, L.-K., and Faeth, G.M., "Structure of Round, Fully-Developed, Buoyant Turbulent Plumes," J. Heat Trans., Vol. 116, 1994, pp. 409-417.

${ }^{13}$ List, E.J., "Turbulent Jets and Plumes," Ann. Rev. Fluid Mech., Vol. 14, 1982, pp. 189-212.

${ }^{14}$ Turner, J.S., Buoyancy Effects in Fluids, Cambridge Univeristy Press, Cambridge, London, 1973.

${ }^{15}$ Turner, J.S., "The Dynamics of Spheroidal Masses of Buoyant Fluid," J. Fluid Mech., Vol. 19, 1964, pp.481490.

${ }^{16}$ Turner, J.S., "Buoyant Plumes and Thermals," $\underline{\text { Ann. }}$ Rev. Fluid Mech., Vol. 1, 1969, pp. 29-44.
${ }^{17}$ Batt, R.G., Brigoni, R.A., and Rowland, D.J., "Temperature-Field Structure Within Atmospheric Buoyant Thermals," J. Fluid Mech., Vol. 141, 1984, pp. 1-25.

${ }^{18}$ Glezer, A., and Coles, D., "An Experimental Study of a Turbulent Vortex Ring," J. Fluid Mech., Vol. 211, 1990, pp. 243-283.

${ }^{19}$ Shariff, K., and Leonard, A., "Vortex Ring," Ann. Rev. Fluid Mech., Vol. 24, 1992, pp. 235-279.

Table 1 Summary of self-preserving buoyant turbulent flows in uniform crossflows. ${ }^{\text {a }}$

\begin{tabular}{|c|c|c|c|c|c|c|c|c|}
\hline Medium & $\left(x_{\mathrm{p}}-x_{\mathrm{o}}\right) / d^{\mathrm{b}}$ & $Q_{\mathrm{o}} /\left(A_{\mathrm{o}} d\right)$ & $\left.x_{\mathrm{o}}\right) / d$ & $R e_{\mathrm{o}}$ & $F r_{\mathrm{o}}$ & $n$ & $C_{\mathrm{x}}$ & $C_{\mathrm{r}}$ \\
\hline \multicolumn{9}{|l|}{ Puffs: } \\
\hline Liquid & 120 & $16-318$ & -23 & $6000-12000$ & --- & $1 / 4$ & 13.0 & 0.14 \\
\hline \multicolumn{9}{|c|}{ Thermals: } \\
\hline Liquid & 200 & $18-318$ & -20 & $4000-11000$ & $14-82$ & $1 / 2$ & 2.0 & 0.14 \\
\hline
\end{tabular}

${ }^{a}$ Both flows satisfied the no-slip convection approximation in the crossflow direction with $y_{\mathrm{o}} / d=0$ and $C_{y}=1$.

${ }^{\mathrm{b}}$ Maximum vertical penetration distance observed. 


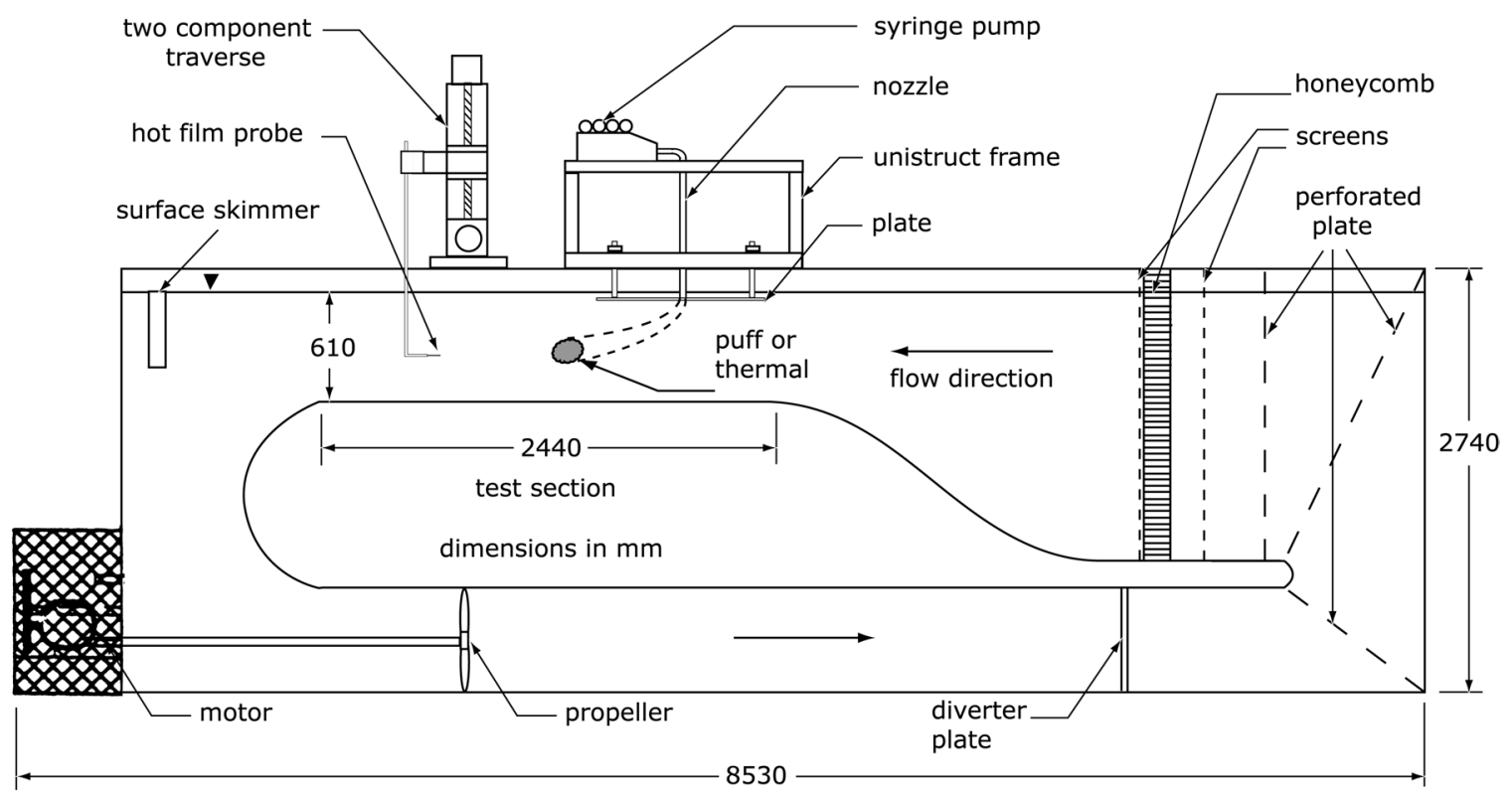

Fig. 1 Sketch of the test apparatus for round nonbuoyant puffs and buoyant thermals in a crossflow.

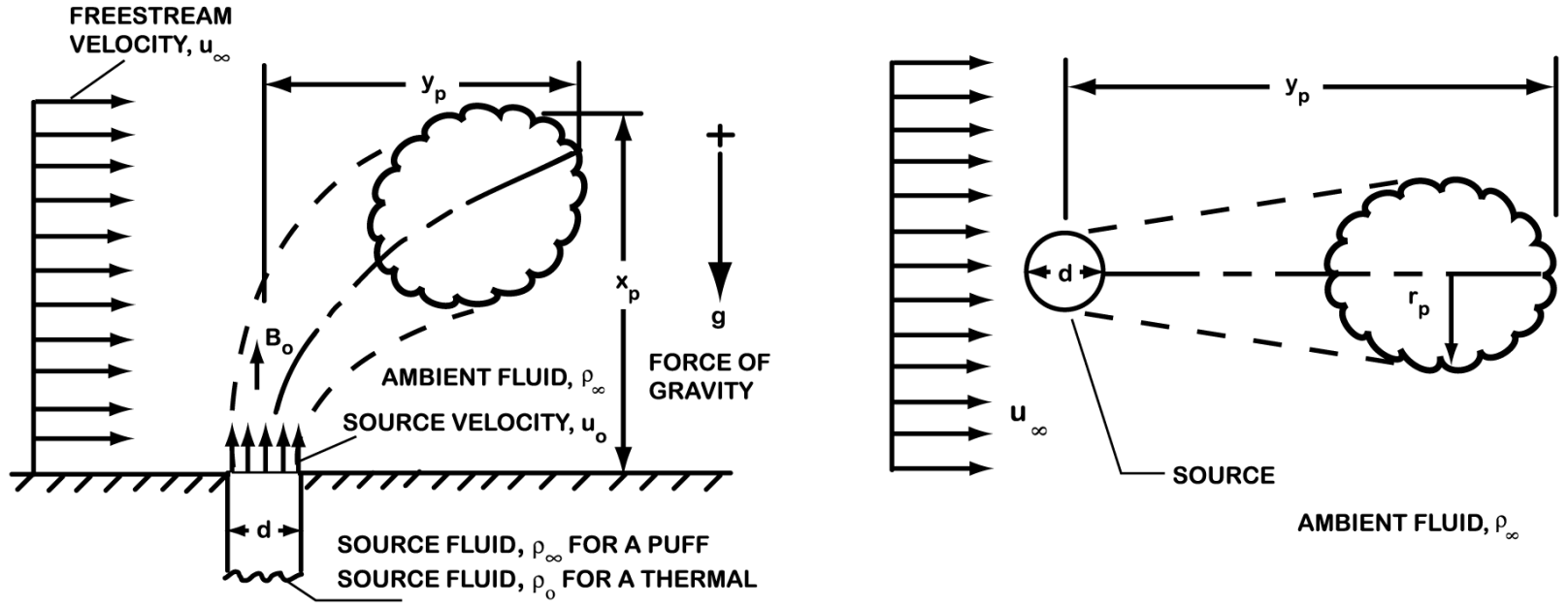

ELEVATION VIEW

PLAN VIEW

Fig. 2 Sketch of round turbulent nonbuoyant puffs and buoyant thermals in a crossflow. 


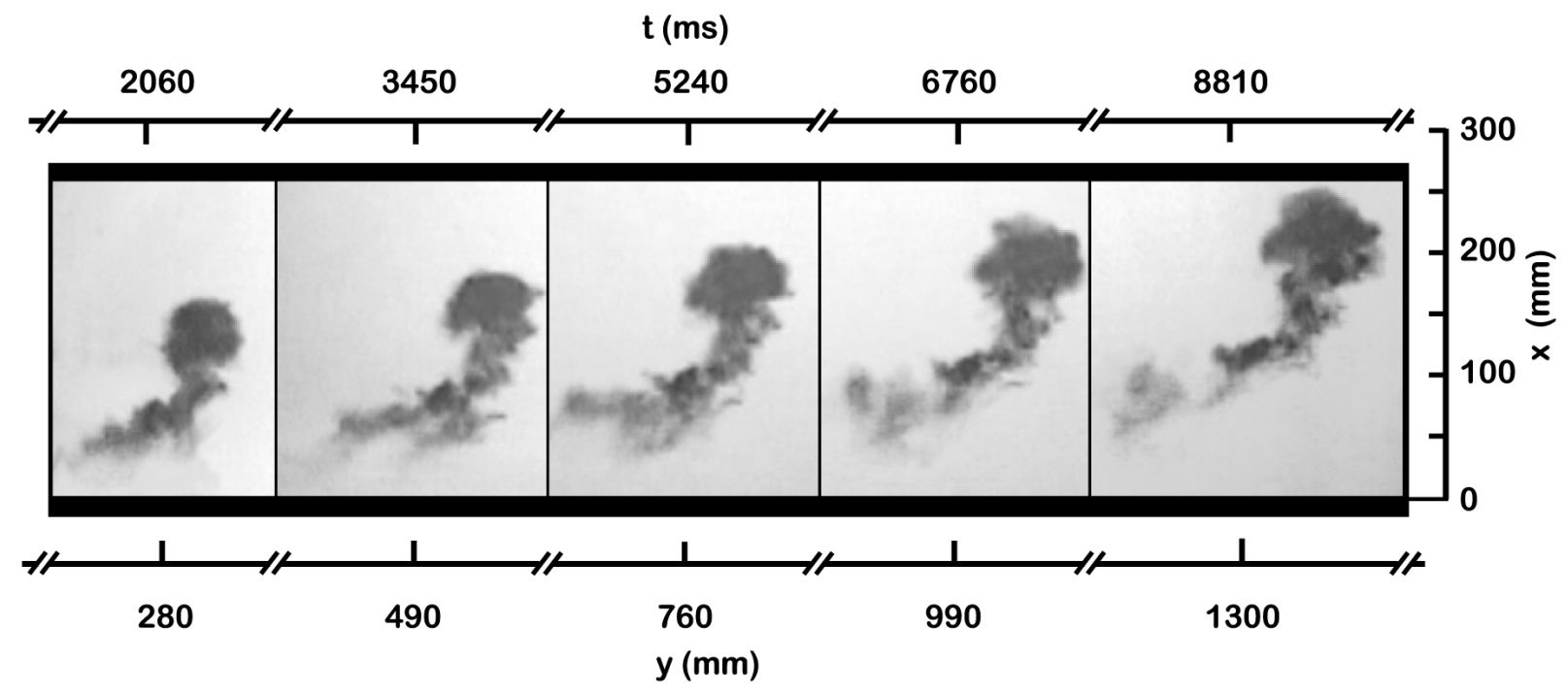

Fig. 3 Visualization of a round turbulent nonbuoyant puff in a uniform crossflow. ( $d=3.2 \mathrm{~mm}, \operatorname{Re}_{\mathrm{o}}=6000$, $\left.Q_{\mathrm{o}} /\left(A_{\mathrm{o}} d\right)=159, \mathrm{u}_{\mathrm{o}} / \mathrm{u}_{\infty}=12\right)$

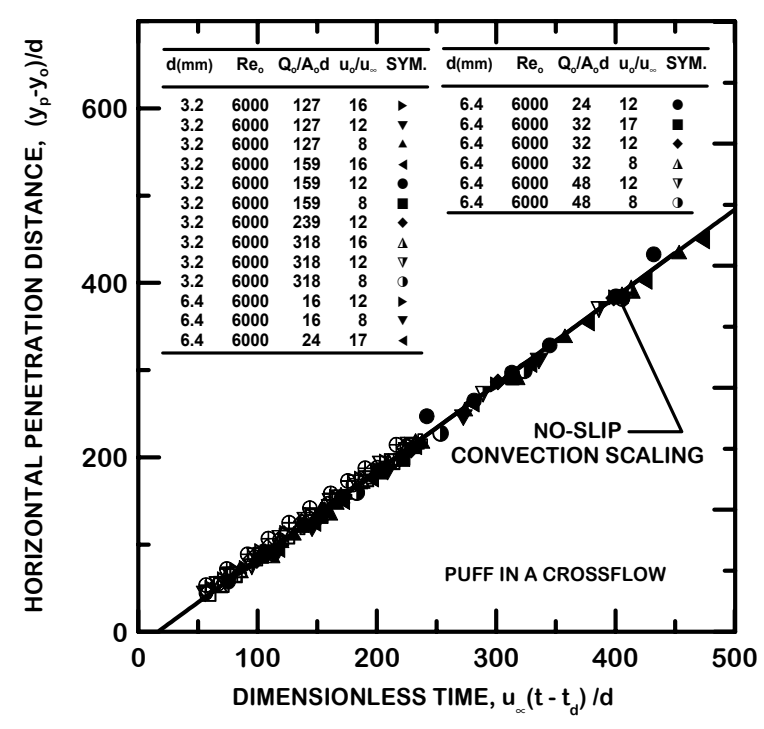

Fig. 4 Horizontal penetration distance as a function of time for round turbulent nonbuoyant puffs.

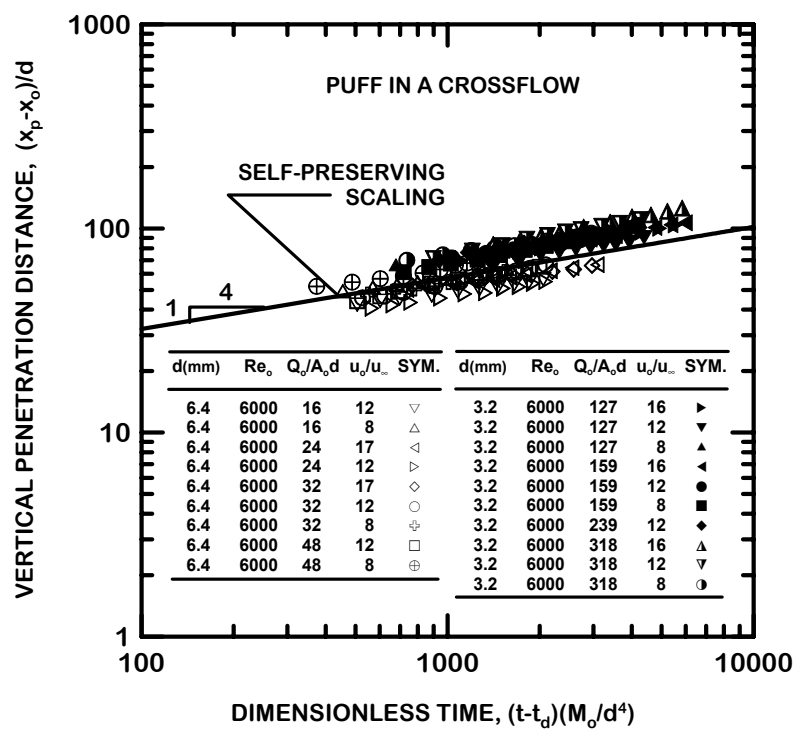

Fig. 5 Vertical penetration distance as a function of time for round turbulent nonbuoyant puffs. 


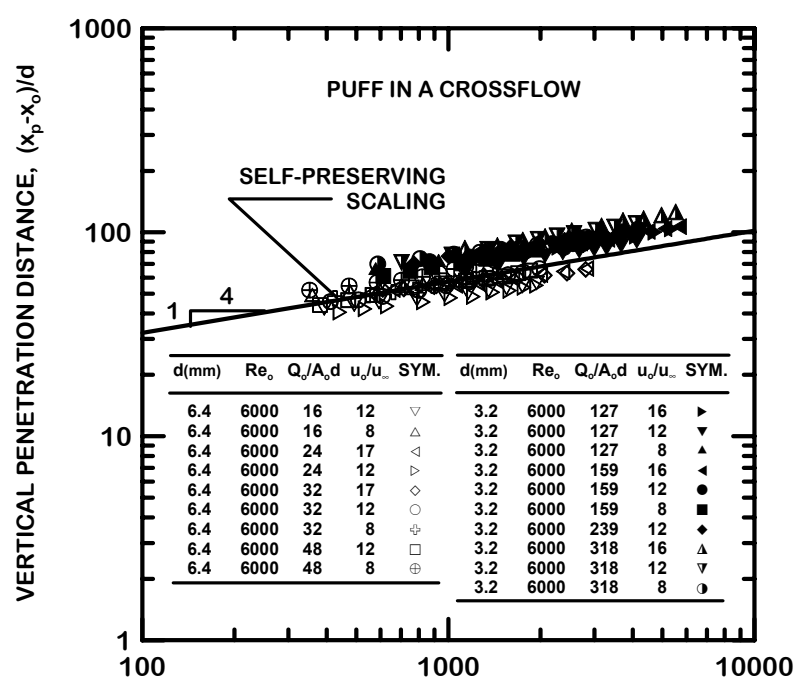

HORIZONTAL PENETRATION DISTANCE, $\left(\left(y_{p}-y_{0}\right) / u_{\alpha}\right)\left(m_{o} / d^{4}\right)$

Fig. 6 Vertical penetration distance as a function of horizontal penetration distance for round turbulent nonbuoyant puffs.

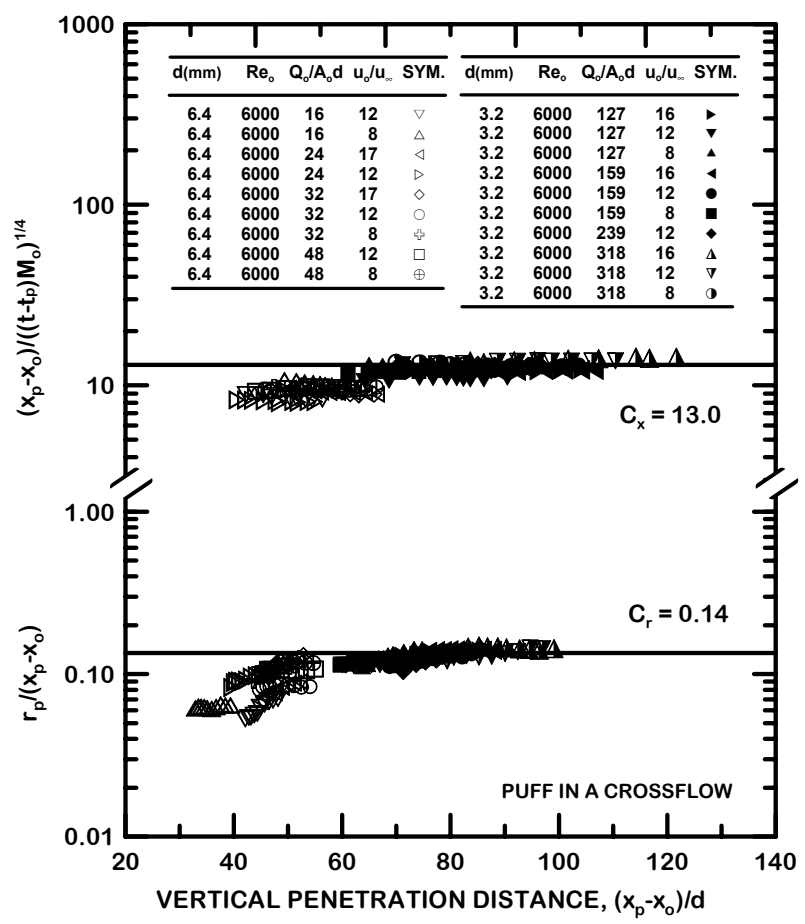

Fig. 7 Radial and vertical penetration distance as a function of vertical penetration distance for puffs in crossflow.

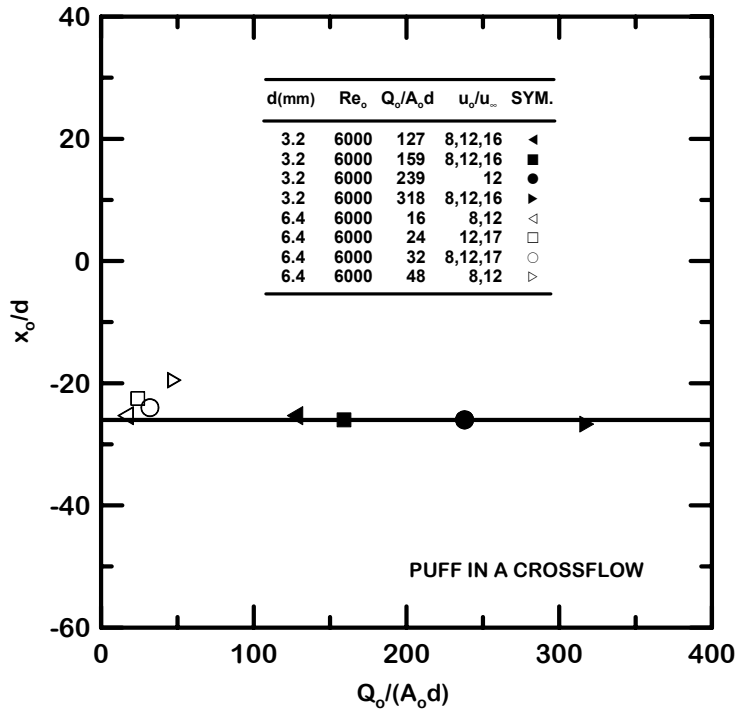

Fig. 8 Virtual origin as a function of volume of injected fluid for round turbulent nonbuoyant puffs. 


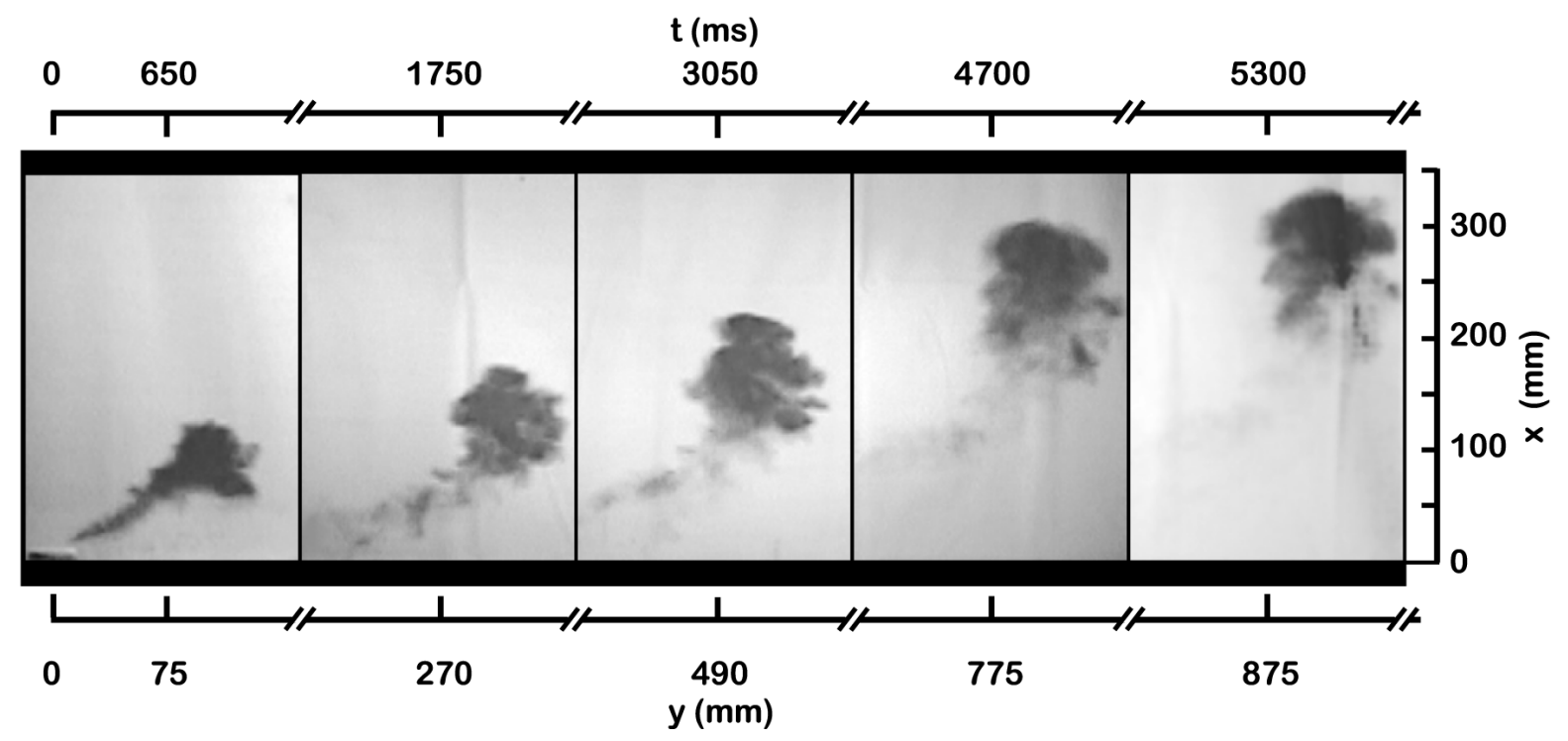

Fig. 9 Visualization of a round turbulent buoyant thermal in a uniform crossflow. ( $d=3.2 \mathrm{~mm}, \operatorname{Re}_{\mathrm{o}}=4000$, $\left.\rho_{\mathrm{o}} / \rho_{\infty}=1.150, Q_{\mathrm{o}} /\left(A_{\mathrm{o}} d\right)=223, \mathrm{Fr}_{\mathrm{o}}=29.3, \mathrm{u}_{\mathrm{o}} / \mathrm{u}_{\infty}=11\right)$

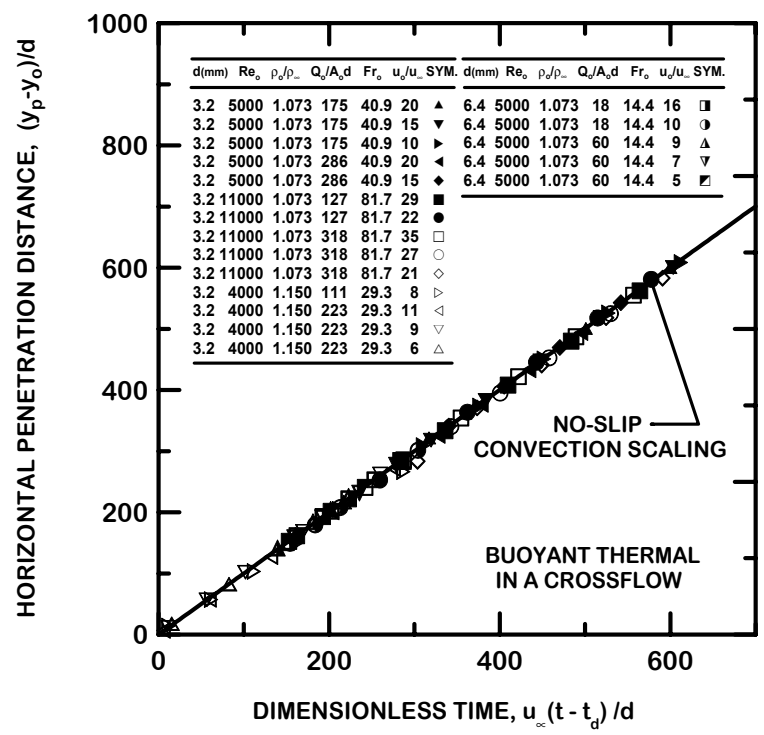

Fig. 10 Horizontal penetration distance as a function of time for round turbulent buoyant thermals.

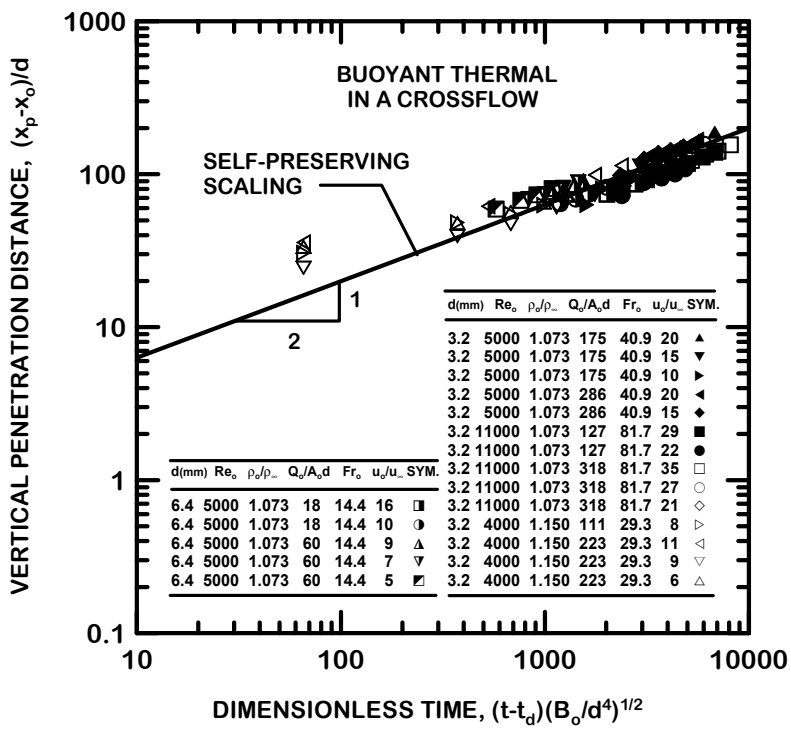

Fig. 11 Vertical penetration distance as a function of time for round turbulent buoyant thermals. 


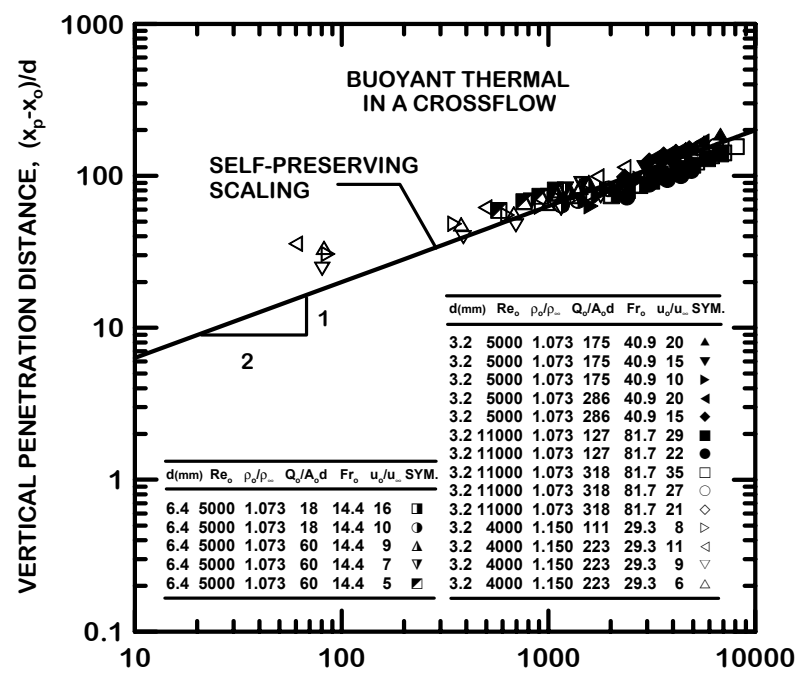

HORIZONTAL PENETRATION DISTANCE, $\left(\left(y_{p}-y_{o}\right) / u_{\propto}\right)\left(B_{o} / d^{4}\right)^{1 / 2}$

Fig. 12 Vertical penetration distance as a function of horizontal penetration distance for round turbulent buoyant thermals.

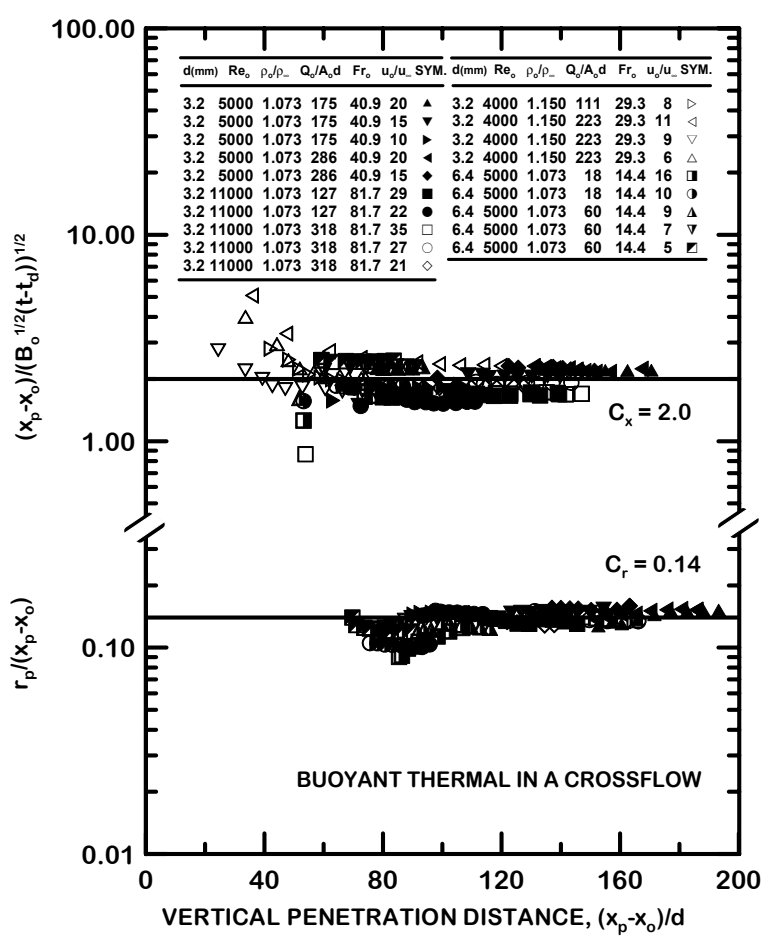

Fig. 13 Radial and vertical penetration distance as a function of vertical penetration distance for thermals in crossflow.

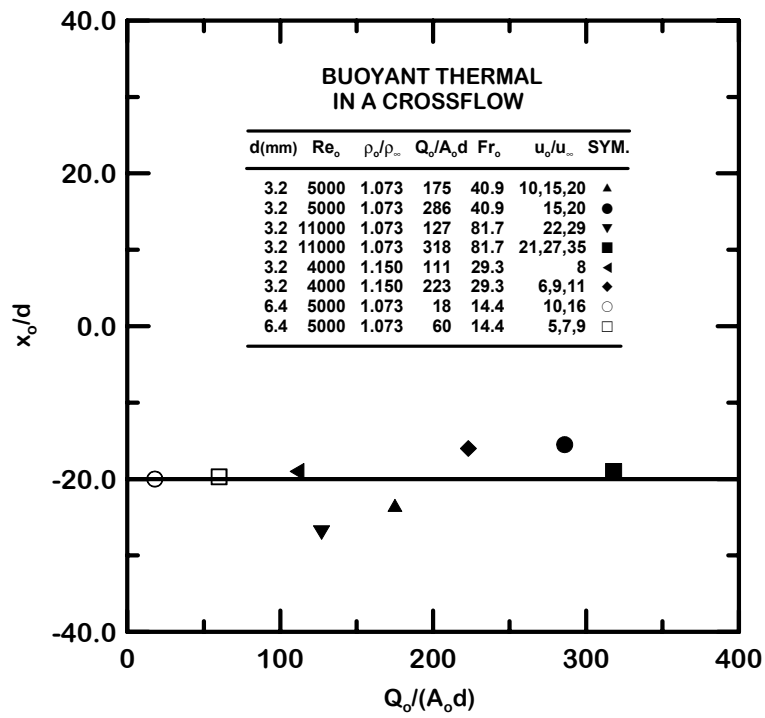

Fig. 14 Virtual origin as a function of volume of injected fluid for round turbulent buoyant thermals. 